\title{
High-momentum tail in the Tonks gas under harmonic confinement
}

\author{
A. Minguzzi, P. Vignolo and M. P. Tosi \\ NEST-INFM and Classe di Scienze, Scuola Normale Superiore, Piazza dei \\ Cavalieri 7, I-56126 Pisa, Italy
}

\begin{abstract}
We use boson-fermion mapping to show that the single-particle momentum distribution in a one-dimensional gas of hard point-like bosons (Tonks gas) inside a harmonic trap decays as $p^{-4}$ at large momentum $p$. The relevant integrals expressing the one-body density matrix are evaluated for small numbers of particles in a simple Monte Carlo approach to test the extent of the asymptotic law and to illustrate the slow decay of correlations between the matter-wave field at different points.
\end{abstract}

Key words: Hard-core Bose gases, Fermi gases, momentum distribution PACS: 05.30.-d, 03.75.Fi

\section{Introduction}

The realization of Bose-Einstein condensates of lower dimensionality in suitable optical and magnetic traps $[1,2]$, and advances in atom waveguide technology with potential applications to atom interferometry and integrated atom optics [3-7], give special motivation to theoretical studies of dilute atomic fluids in a regime where the quantum dynamics becomes quasi-one-dimensional (1D). The condensate fraction is depleted in this situation even at zero temperature, and the standard stationary or time-dependent Gross-Pitaevskii theory loses its usefulness [8-10]. An extreme limiting example is provided by the so-called Tonks gas of hard point-like bosons, in which the transverse confinement is taken to be so strong that the dynamics reduces to strictly 1D motion. The occupation of the lowest orbital behaves in this case with the number $N$ of particles as $N^{n}$ with $n<1$ [11]. The momentum distribution still has a strong peak on approaching zero momentum [8,11], and coherence effects have been shown to be present in the form of Talbot recurrences following an optical lattice pulse [12] and of dark soliton-like behaviour in response to a phase-imprinting pulse [13].

Preprint submitted to Elsevier Preprint 26 October 2018 
A number of properties of the Tonks gas of impenetrable bosons can be calculated exactly through a mapping into an ideal 1D gas of spin-polarized fermions [14]. In both fluids each particle behaves as a hard point which is at any time free to move on a segment comprised by its two immediate neighbours and can exchange with either of them on contact. Considering in particular the many-body ground state, the boson-fermion mapping consists of the identity between the bosonic wave function and the modulus of the fermionic wave function [14]. This of course implies that, whereas all configurational probability distributions are the same in the two fluids, their single-particle momentum distributions are in general completely different [15].

A visualization of the ground state can be obtained by varying the position of one particle while all others are kept fixed. The fermionic wave function varies smoothly from positive to negative across each node so as to mimimize the kinetic energy, and hence the bosonic wave function, being everywhere non-negative, has a cusp at each node. We show in this paper that from this cusp condition the momentum distribution of the Tonks gas under harmonic confinement acquires a long tail at high momentum $p$, decaying asymptotically as $p^{-4}$. We also gauge the extent of this asymptotic behaviour by Monte Carlo calculations of the momentum distribution for the case of two particles in a harmonic well, where high statistical accuracy and simple analytical results are easily obtained.

\section{Asymptotic tails of the bosonic momentum distribution}

The single-particle momentum distribution $n(p)$ at momentum $p=\hbar k$ is the Fourier transform of the one-body density matrix $\rho\left(x, x^{\prime}\right)$ with respect to the relative coordinate $r=x-x^{\prime}$, averaged over the centre-of-mass coordinate $R=\left(x+x^{\prime}\right) / 2$. The ground-state wave function for a Tonks gas of $N$ bosons under longitudinal harmonic confinement has been calculated by Girardeau et al. [11], with the result

$$
\psi_{0}^{B}\left(x_{1}, \ldots, x_{N}\right)=C_{N} \prod_{1 \leq j<k \leq N}\left|x_{k}-x_{j}\right| \prod_{i=1}^{N} \exp \left(-x_{j}^{2} / 2 x_{h o}^{2}\right) .
$$

Here, $C_{N}$ is a normalization constant and $x_{h o}=(\hbar / m \omega)^{1 / 2}$ is the harmonic oscillator length determined by the trap frequency $\omega$ and the particle mass $m$. Taking $x_{h o}$ as the unit of length, the momentum distribution is then given by

$$
n(p)=\mathcal{N}_{N} \int_{-\infty}^{+\infty} d R \int_{-\infty}^{+\infty} d r \exp \left[-i k r-\left(R^{2}+r^{2} / 4\right)\right] \int_{-\infty}^{+\infty} d Q_{2} \ldots \int_{-\infty}^{+\infty} d Q_{N}
$$




$$
\times \exp \left(-\sum_{l=2}^{N} Q_{l}^{2}\right)\left\{\left[\prod_{2 \leq j<k \leq N}\left(Q_{k}-Q_{j}\right)^{2}\right] \prod_{i=2}^{N}\left|\left(Q_{i}-R\right)^{2}-r^{2} / 4\right|\right\}(2)
$$

where $\mathcal{N}_{N}$ is a factor depending on the number of particles.

Let us first treat the case $N=2$. The momentum distribution is given by

$$
n(p)=\mathcal{N}_{2} \int_{-\infty}^{+\infty} d R \int_{-\infty}^{+\infty} d Q_{2} \exp \left(-R^{2}-Q_{2}^{2}\right) I\left(Q_{2}, R ; k\right)
$$

where

$$
I\left(Q_{2}, R ; k\right)=\int_{-\infty}^{+\infty} d r\left|\left(Q_{2}-R\right)^{2}-r^{2} / 4\right| \exp \left(-i k r-r^{2} / 4\right) .
$$

The integrand has two singularities of the type $|r \pm \alpha|$ and we can use a theorem given in the book of Lighthill [16] to evaluate its asymptotic value in the limit $k \rightarrow \infty$. Using the result $\int d z|z| \exp (i k z)=-2 / k^{2}$ we get

$$
\lim _{k \rightarrow \infty} I\left(Q_{2}, R ; k\right)=-\frac{2\left|Q_{2}-R\right|}{k^{2}} \cos \left[2 k\left(Q_{2}-R\right)\right] \exp \left[-\left(Q_{2}-R\right)^{2}\right] .
$$

Introducing the notations $X=\left(Q_{2}-R\right) / \sqrt{2}$ and $Y=\left(Q_{2}+R\right) / \sqrt{2}$, the asymptotic behaviour of the momentum distribution is then given by

$$
\lim _{p \rightarrow \infty} n(p)=\frac{-2 \sqrt{2}}{k^{2}} \mathcal{N}_{2} \int_{-\infty}^{+\infty} d Y \exp \left(-Y^{2}\right) \int_{-\infty}^{+\infty} d X|X| \cos (2 \sqrt{2} k X) \exp \left(-3 X^{2}\right)(6)
$$

and Lighthill's theorem can again be employed to evaluate the integral over $X$. The final result is

$$
\lim _{p \rightarrow \infty} n(p)=2 \sqrt{\frac{2}{\pi}} \frac{(\hbar m \omega)^{-3 / 2}}{p^{4}}
$$

having used the value of the normalization constant $C_{2}$ reported by Girardeau et al. [11].

In applying the above procedure to the calculation of the asymptotic value of Eq. (2) for arbitrary $N$, we notice that the same pair of singularities arises for each value of the index $i$ and that they all contribute equally to the final 
result. Choosing $i=2$, we thus find

$$
\lim _{p \rightarrow \infty} n(p)=\frac{A_{N}}{p^{4}}
$$

where the coefficient $A_{N}$ is determined by a multiple integral,

$$
\begin{aligned}
A_{N} & \propto-(N-1) \mathcal{N}_{N} \int_{-\infty}^{+\infty} d Y \exp \left(-Y^{2}\right) \int_{-\infty}^{+\infty} d Q_{3} \ldots \int_{-\infty}^{+\infty} d Q_{N} \exp \left(-\sum_{l=3}^{N} Q_{l}^{2}\right) \\
& \times\left[\prod_{3 \leq j<k \leq N}\left(Q_{k}-Q_{j}\right)^{2}\left(Q_{k}-Y / \sqrt{2}\right)\left(Q_{j}-Y / \sqrt{2}\right)\right] .
\end{aligned}
$$

We have thus shown that the momentum distribution of the Tonks gas in a harmonic trap decays asymptotically as the inverse fourth power of the momentum.

\section{Numerical results by a Monte Carlo method}

In this section we evaluate the one-body density matrix and the momentum distribution function for a small number of particles by a simple Monte Carlo method. The density matrix involves the integrals over $\left(Q_{2}, \ldots, Q_{N}\right)$ entering the second line in Eq. (2). These are calculated by (i) extracting for each variable $Q_{i}$ a random number distributed along a Gaussian of unitary width, and (ii) averaging over all trials the corresponding values of the factor contained in the curly brackets in the integrand. The two further integrals that are required for the evaluation of $n(p)$ are calculated by a similar procedure. A more refined numerical method would be needed to obtain high statistical accuracy for a number of particles $N>5$.

We report in the following our numerical results for $N=2$. Figure 1 shows the function $\rho(x, 0)$ as a function of $x$ for a pair of hard bosons, after suppression of the Gaussian decay imposed by the confinement through multiplication by $[\rho(x) \rho(0)]^{-1 / 2}$ where $\rho(x)$ is the particle density. The comparison with the same function for a pair of non-interacting fermions, for which a direct analytic calculation yields $\rho(x, 0) /[\rho(x) \rho(0)]^{1 / 2}=\left(1+2 x^{2}\right)^{-1 / 2}$, illustrates the role of statistics in determining the rate of decay of the density matrix. It may be remarked that the density matrix for the Bose gas reflects its spatial coherence and can be determined experimentally by measuring the interference pattern of two matter-wave beams emitted from two spatially separated regions of the trap [17]. 
Figure 2 reports the corresponding results that we obtain for the momentum distribution $n(p)$ in the case of two Bose or Fermi particles. The analytical result for fermions, with $n(p)$ described by the function $\pi^{-1 / 2}\left(1+2 y^{2}\right) \exp \left(-y^{2}\right)$ where $y=p /(\hbar m \omega)^{1 / 2}$, is shown by the full line and serves to illustrate the accuracy of the Monte Carlo calculation.

Finally, Figure 3 tests the range of applicability of the asymptotic law given in Eq. (7) by reporting it in a log-log plot against the momentum distribution obtained in two Monte Carlo runs for a pair of hard bosons.

\section{Concluding remarks}

In this work we have demonstrated two aspects of the correlations which arise from Bose statistics in a 1D gas of hard, point-like bosons inside a harmonic trap. A very remarkable feature of this system is the extremely slow decay of the high-momentum tail in the single-particle momentum distribution, which we have determined analytically and verified numerically. We have also shown that the one-body density matrix decays very slowly, after elimination of the Gaussian factors that are imposed by the trap.

A consequence of the $p^{-4}$ tail in the momentum distribution is that the mean square fluctuation of the kinetic energy of the Tonks gas, which involves the fourth moment of $n(p)$, diverges under harmonic confinement. This property is even more remarkable if one considers that the kinetic energy density of the Tonks gas is the same as that of its fermionic map. This latter result, which can be demonstrated analytically in full detail from the bosonic and fermionic ground-state wave functions [18], may be viewed most directly to be a consequence of the basic theorem of density functional theory: the kinetic

energy density is a unique functional of the particle density, which remains unchanged in the boson-fermion mapping.

We acknowledge support from INFM through PRA2001.

\section{References}

[1] A. Görlitz, J. M. Vogels, A. E. Leanhardt, C. Raman, T. L. Gustavson, J. R. Abo-Shaeer, A. P. Chikkatur, S. Gupta, S. Inouye and W. Ketterle, Phys. Rev. Lett. 87 (2001) 130402.

[2] M. Greiner, I. Bloch, O. Mandel, T. W. Hänsch and T. Esslinger, Phys. Rev. Lett. 87 (2001) 160405. 
[3] J. H. Thywissen, R. M. Westervelt and M. Prentiss, Phys. Rev. Lett. 83 (1999) 3762 .

[4] D. Müller, D. Z. Anderson, R. J. Grow, P. D. D. Schwindt and E. A. Cornell, Phys. Rev. Lett. 83 (1999) 5194.

[5] N. H. Dekker, C. S. Lee, V. Lorent, J. H. Thywissen, S. P. Smith, M. Drndic, R. M. Westervelt and M. Prentiss, Phys. Rev. Lett. 84 (2000) 1124.

[6] M. Key, J. G. Hughes, W. Rooijakkers, B. E. Sauer and E. A. Hinds, Phys. Rev. Lett. 84 (2000) 1371.

[7] K. Bongs, S. Burger, S. Dettmer, D. Hellweg, J. Arlt, W. Ertmer and K. Sengstock, Phys. Rev. A 63 (2001) 031602.

[8] M. Olshanii, Phys. Rev. Lett. 81 (1998) 938.

[9] D. S. Petrov, G. V. Shlyapnikov and J. T. M. Walraven, Phys. Rev. Lett. 85 (2000) 3745.

[10] M. D. Girardeau and E. M. Wright, Phys. Rev. Lett. 87 (2001) 210401.

[11] M. D. Girardeau, E. M. Wright and J. M. Triscari, Phys. Rev. A 63 (2001) 033601.

[12] A. G. Rojo, G. L. Cohen and P. R. Berman, Phys. Rev. A 60 (1999) 1482.

[13] M. D. Girardeau and E. M. Wright, Phys. Rev. Lett. 84 (2000) 5691.

[14] M. Girardeau, J. Math. Phys. 1 (1960) 516.

[15] M. D. Girardeau and E. M. Wright, Phys. Rev. Lett. 87 (2001) 050403.

[16] M. J. Lighthill, Introduction to Fourier analysis and generalised functions (Cambridge University Press, Cambridge 1959).

[17] I. Bloch, T. W. Hänsch and T. Esslinger, Nature 403 (2000) 166.

[18] P. Vignolo, A. Minguzzi and M. P. Tosi, to be published. 


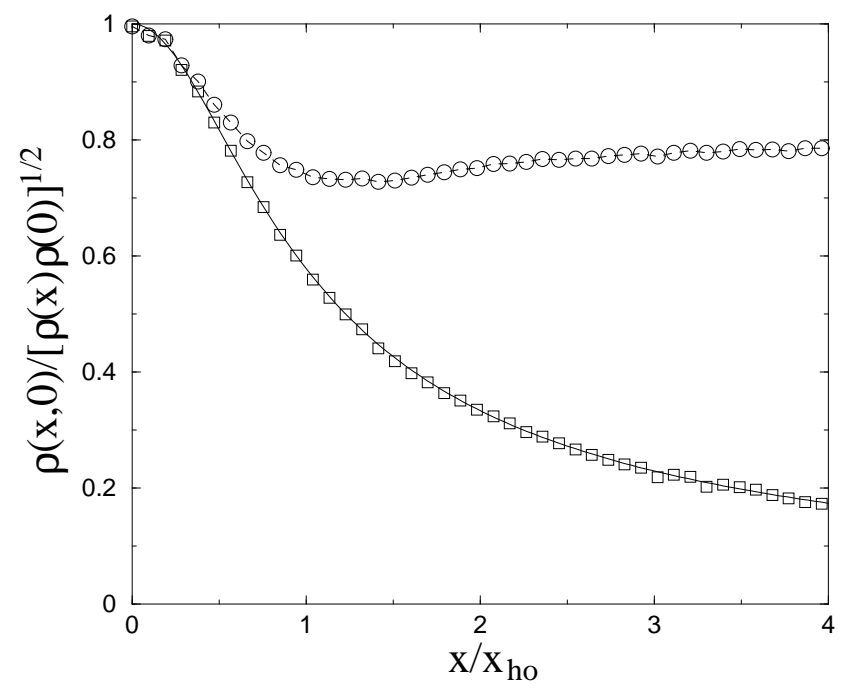

Fig. 1. Monte Carlo results for the one-body density matrix $\rho\left(x, x^{\prime}\right) /\left[\rho(x) \rho\left(x^{\prime}\right)\right]^{1 / 2}$, calculated as a function of $x$ at $x^{\prime}=0$, for a pair of hard point-like bosons (circles) and a pair of non-interacting fermions (squares) moving in 1D under harmonic confinement. The full line gives the exact analytical result for the case of fermions. The error bars in the Monte Carlo data are within the size of the symbols.

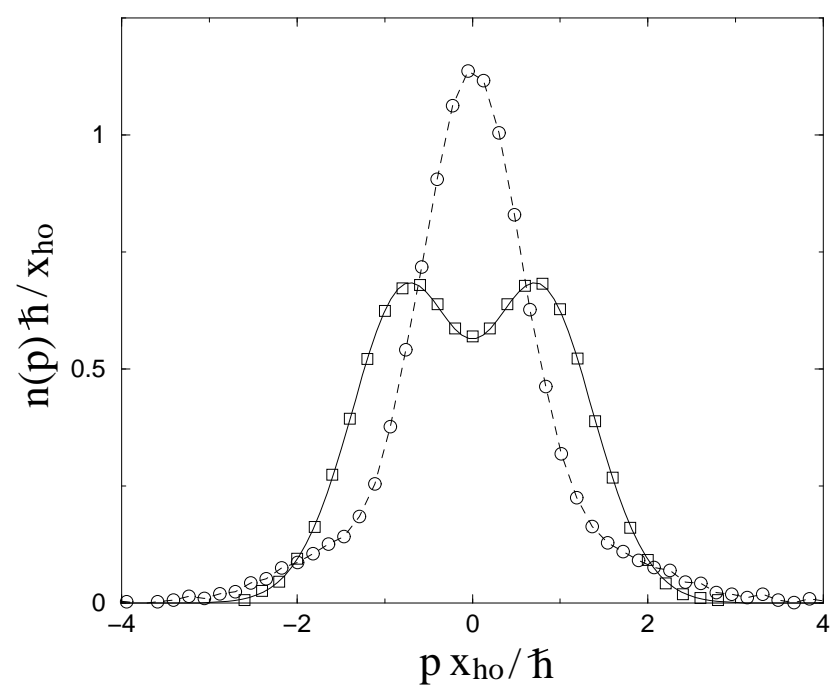

Fig. 2. Monte Carlo results for the momentum distribution of a pair of hard point-like bosons (circles) and a pair of non-interacting fermions (squares) moving in 1D under harmonic confinement. The full line gives the exact analytical result for the case of fermions. The error bars in the Monte Carlo data are within the size of the symbols. 


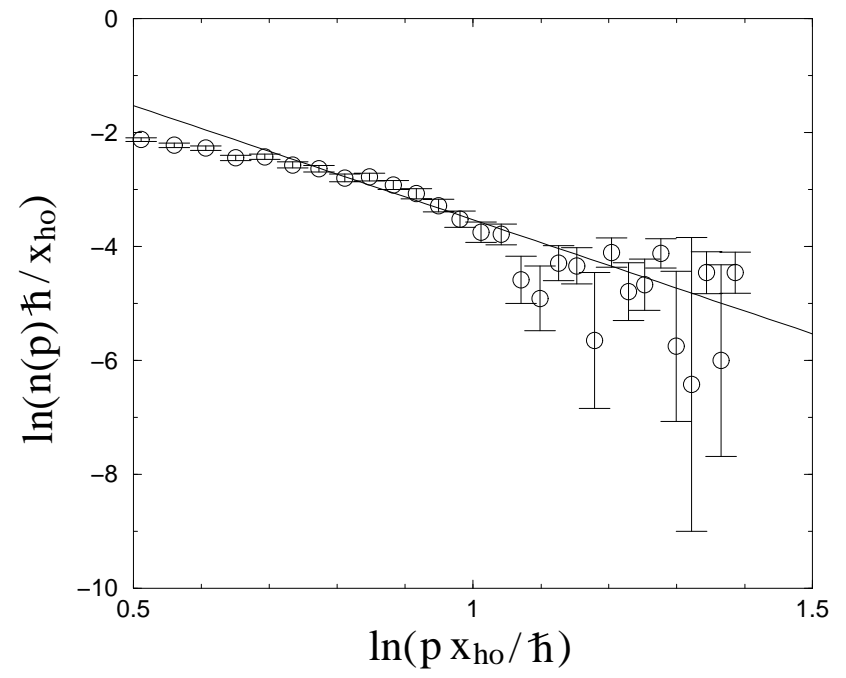

Fig. 3. Log-log plot of the momentum distribution of a pair of hard point-like bosons moving in 1D under harmonic confinement, from Monte Carlo integration (circles with error bars). The full line shows the asymptotic law given in Eq. (7). 